\title{
RELAÇÃO DAS OBRAS ENTRADAS NA BIBLIO. TECA DA FACULDADE DE DIREITO DE SÃO PAULO, NO PERIODO COMPREENDIDO ENTRE 16 DE NOVEMBRO DE 1935 E DE ABRIL DE 1936:
}

\section{OBRAS GERAIS (0)}

Actas da Camara da Cidade de São Paulo-1835-Vol XXVIII-São Paulo, 1935-Broch.-1 vol.-Permuta.

Actas da Camara Municipal de São Paulo-1744-1836-São Paulo, 19181923-Broch.-19 vols.-Permuta.

Adolpho Augusto Pinto-A Cathedral de São Paulo-São Paulo, 1930Broch.-1 vol.-Doação.

Almanaque do Estado do Ceará-Fortaleza, 1936,-Broch.-1 vol.-Doação. da Imprensa Official do Estado do Ceará.

Anales del Instituto Nacional de Prevision-Vol. XXVII-Madrid, 1935Broch.-1 vol.-Permuta.

Annaes da Assembléa Nacional Constituinte-Rio de Janeiro, 1934-1935 -Broch.-8 vols.-Doação da Secrecretaria da Camara dos Deputados Federal.

Annaes da Escola de Minas de OuroPreto-N..$^{\circ}$ 26-Ouro Preto, 1935Broch.-1 vol.-Permuta.

Annexos ao Relatorio n. ${ }^{\circ}$ 82, da Companhia Mogyana de Estradas de Ferro para a Assembléa Geral de 27 de Maio de 1935 e correspondentes, ao anno de 1934-Campinas, 1935Broch.-1 vol.-Doação do Dr. José Carlos de Macedo Soares.
Annual English Supplement of The Asahi Osaka and Tokyo-Osaka, 1934 -Encad.--1 vol.-Doação.

Annuario da Escola Polytechnica para o anno de 1935-São Paulo, 1935Broch.-vol.-Permuta

Annuario da Faculdade de Direito do Recife-Anno lectivo de 1935-Recife, 1935-Broch.-vol.-Permuta

Annuario per l'anno academico 1932-33 e 1933-34-R. Universitá Degli Studi di Padova-Padova, 1934-Broch.2 vols.-Permuta

Annuario per l'anno scolastico 1933-34 -Regia Università Degli Studi di Cagliari-Cagliari, 1933-Broch.-1 vol.-Permuta

Anuario de la Academia Colombiana de Jurisprudencia-vol. I-1933-Bogotá-Colombia, 1934-Broch.-1 vol. -Permuta

Archivos do Instituto Biologico-Vol. VI-São Paulo, 1935-Broch.— vol. -Permuta

Archivos do .Museu Nacional-vol. XXXV-Rio de Janeiro, 1933-Broch. -1 vol.-Permuta.

Boletim Commercial-Diario OfficialRio de Janeiro, 1935-Broch.-1 vol. -Compra

Boletim da Associação dos Proprietarios de São Paulo-São Paulo, 1933 -1934-Broch.-2 vols.-Duação da mesma. 
Boletim da Bibliotheca da Universidade de Coimbra-Vol. XI e supplemento ao vol. XI-Coimbra, 1934Broch.-1 vol.--Permuta.

Boletim da Directoria Technica de Educação-Pernambuco, 1931/1934Broch.-4 vols.-Permuta

Boletim da Faculdade de Direito da Universidade de Coimbra-Vols. VI, VII, VIII, IX, X, XI e XIII-Coimbra, $1920,1921,1926,1934$-Broch. -8 vols. -Permuta

Boletim da União Pan-AmericanaWashington, 1935-Broch.-1 vol.Permuta.

Boletim do Instituto de EngenhariaVol. XXII-São Paulo, 1935-Broch. -1 vol.-Permuta.

Boletim do Ministerio do Trabalho, Industria e Commercio-N. ${ }^{\circ}$ s 14,15 , 16, 18, 19-Rio de Janeiro, 1935/1936 Brochs. -5 vols.-Permuta.

Boletim do Museu Nacional-Vol. XRio de Janeiro, 1934-Broch.-vol. Permuta.

Catalogo de productos do AmazonasServiço de propaganda da Associação Commercial-Broch.--1 vol.Permuta.

Diario da Justiça-Rio de Janeiro, 1935/1936-Encad.--7 vols.-Compra.

Diario Official da Republica dos Estados Unidos do Brasil-Rio de Janeiro, 1935/1936-Eincad.-11 vols.Compra

Diario Official do Estado de São Paulo -São paulo, 1935/1936-Encad.-5 vols.-Doação.

Emilio Guimarãaes-Brasil-Accordãos (Repertorio de Jurisprudencia dos Tribunaes Brasileiros)-Vols. IV, V, VI, VII e VIII-Rio de Janeiro, 1935/1936-Encads.-5 vols.-Compra.

Gallo (Alfonso)-Le malatie del libro le cure ed i restauri-Milano-Encad. -1 vol.-Compra.

Georg Wissowa, Wilhelm Kroll e Karl Mittelhans-Realengyklopädie Der Klassicchen Altertums Wissenschaft -Sttutgart-Broch.-1 vol.-Doação do Dr. Prof. Hans Kreller.
Guia Fiscal-Orgão da Associação dos Collectores e Escrivães Federaes do Estado de São Paulo-São Paulo, 1935-Broch.-1 vol.-Permuta.

Guia para fichado y catalogación-Tabla de materias y palabras-claveBiblioteca Nacional, Buenos Aires, 1935-Broch.-1 vol.Permuta.

Idort-Orgão do Instituto de Organização Racional do Trabalho-São Paulo, 1935-Broch._1 vol.-Permuta.

Intelligencia-Mensario da opinião mundial-São Paulo, 1935-Broch.12 vols.-Permuta.

José Fernandes Moreno-Indice alphabetico e remissivo da Constituição Estadual-São Paulo, 1935-Broch.1 vol.-Doação do autor

Jornal des Assurances-Incendie, Vie, Maritimes, Accidentes, etc.-Paris, 1914/1915-Broch.-12 vols.-Doação.

Jurisprudencia-Boletin del Instituto de Enseñanza Practica-Facultad de Derecho y Ciencias Sociales-Universidad de Buenos Aires-Buenos Aires, 1935-Broch--1 vol.-Permuta.

La Biblioteca Nacional en 1934Memoria elevada al Exmo. Señor Ministro de Justicia y Instrucción Pública Dr. D. Manuel M. de Yriondo-Buenos Aires, 1935-Broch.-1 vol.-Permuta.

La Revista Economica SudamericanaOrgano de la Union Industrial Uruguaya-Montevideo, 1935-Broch.-1 vol.-Permuta.

La Vie Intellectuelle-TomeXL-Juvisy' 1936-Broch.-1 vol.-Permuta.

L'École Libre $\mathrm{de}_{\mathbf{s}}$ Sciences Politiques -Paris, 1935-Broch.-1 vol.-Permuta.

Le Mois-Synthèse de l'activité mondiale-Vols. 53, 54, 55, 56, 57, 58, 59, $60,61,62$ e 63-Paris, 1935/1936Broch.-11 vols.-Compra.

London \& Cambridge Economic Service-London, 1935-Broch.-2 vols.-

Boletim Commercial-Diario OfficiuL'Université de Toulose fondée en 1929 La Ville-La Région-Annuaire 1934 -Broch.-1 vol. Permuta. 
Memoria del Departamento y ejecutivo 1933/1934-Municipalidad de la Ciudad de Buenos Aires-Buenos Aires, 1935-Broch.-1 vol.-Permuta.

Mendonça Lima-As necessidades da Estrada de Ferro Central do Brasil -Rio de Janeiro, 1935-Broch.-1 vol.-Doação.

Mensario de Estatistica da Producção -Publicação da Directoria de Estatistica da Producção do Ministerio da Agricultura-Anno I-Rio de Janeiro, 1935-Broch.-1 vol.-Permuta.

Michigan Law Review-Vol.XXXIIIMichigan, 1935-Broch.-1 vol.Permuta.

Niemeyer zeitschrift für Internationales Recht-Band XLIX-L-Berlim, 1934/1935-Broch.-2 vols.-Permuta.

Nouvelle Revue de Hongrie-Tomme LIII-Budapest, 1935-Broch.-1 vol. -Permuta.

Otlet (Paul)-Traité de documentation le livre sur le livre-Bruxellas, 1934 -Broch-1 vol.-Compra.

Registro geral da Camara Manicipal de São Paulo-1583-1831-São Paulo, 1917/1922-Broch.-18 vols.-Permuta.

Registro Geral da Camara Municipal de São Paulo-1830/1831-Vol. XXISão Paulo, 1936-Broch.-1 vol.Permuta.

Relatorio apresentado ao Snr. Ministro da Justiça e Negocios Interiores pelo Procurador Geral do Districto Federal-Rio de Janeiro, 1935Broch.-1 vol.-Doação.

Relatorio balanço e contas do Jockey Club de São Paulo, anno de 1934Sáo Paulo, 1935--Broch.--1 vol.Doação do Dr. José Carlos de Macedo Soares.

Relatorio da Directoria do Automovel Club do Brasil, apresentado á Assembléa geral ordinaria de maio de 1934-Rio de Janeiro-Broch.-1 vol. Doação do Dr. José Carlos de Macedo Soares.

Relatorio da Irmandade da Santa Casa de Misericordia de São Paulo do anno de 1934-São Paulo, 1935Broch.-1 vol.-Doação do Dr. Antonio de Padua Salles.

Relatorio da Prefeitura Municipal de São Bernardo, apresentado ao Dr. Domicio Pacheco e Silva, director do D. A. M. pelo Prefeito Dr Felicio Laurito-São Paulo, 1935-Broch.-1 vol.-Doação da Prefeitura Municipal de São Paulo.

Relatorio da Procuradoria Judicial da Fazenda do Estado-São Paulo, 1936 -Broch.-1 vol.-Doação da mesma.

Relatorio e contas do anno de 1933 , do Automovel Club de São Paulo, apresentados á Assembléa geral ordinaria de 28 de março de 1934São Paulo, 1934-Broch.— vol.Doação do Dr. José Carlos de Macedo Soares.

Répertoire du Journal des Assurances -Paris, 1900/1914-Broch.-2 vols.Doação.

Repertorio de Jurisprudencia-Vol. I -São Paulo, 1908-Broch.-1 vol._ Doação.

Revista Academica da Faculdade de Direito de Recife-Vol. XLIII-Pernambuco, 1935-Brochs.-2 vols.Permuta.

Revista Academica Brasileira de Letras -Vols. XLVIII-XLIX-Rio de Janeiro, 1935 -Brochs.-2 vols.-Permuta.

Revista da Educação-Orgão do Departamento de Instrucção Publica do Estado da Bahia -Vol. I-Bahia, 1933/1935-Broch.-1 vol.-Permuta.

Revista da Faculdade de Direito da Bahia-Vol. X-Bahia, 1935-Broch. -1 vol.-Permuta.

Revista da Universidade de Minas Geraes-Vol. III-Rio de Janeiro, 1935 -Broch-1 vol.-Permuta.

Revista de Criminologia, Psiquiatria y Medicina Legal-Vol. XXII-Buenos Aires, 1935-Broch.-1 vol.-Permuta.

Revista de Critica Jadiciaria-Vol. XXI Rio de Janeiro, 1935-Broch.-1 vol. -Compra.

Revista de Cultura-Anno IX-Rio do Janeiro, 1935 -Broch -1 vol.-Permuta. 
Revista de Derecho y Administración Municipal-Buenos Aires, 1935-Broch. -12 vols.-Permuta.

Revista de Direito Civil Commercial e Criminal-Vol. CXV-Rio de Janeiro, 1935-Encad.-1 vol-Compra.

Revista de Direito $\mathbf{P}^{\mathbf{e}}$ nal-Orgão Official da Sociedade Brasileira de Criminologia-Vols. X e XI-Ria de Janeiro, 1933/1935-Brochs_-2 vols. -Permuta.

Revista de Jurisprudencia BrasileiraVols. XXVIII, XXIX e XXX-Rio de Janeiro, 1935/1936-Brochs. -3 vols. -Permuta.

Revista de la Caja de Socorros de la Policia y Bomberos de la CapitalBuenos Aires, 1935-Broch.-1 vol. -Permuta.

Revista de Mutualismo Escolar y Previsión Infantil-Madrid, 1935-Broch. -1 vol_-Permuta.

Revista de Neurologia e Psychiatria de São Paulo-Vol. I-São Paulo, 1934/1935-Broch.-1 vol.-Permuta.

Revista de Propriedade Industrial-Rio de Janeiro, 1935-Broch.-1 vol.Compra.

Revista del Colegio de Abogados de Buenos Air $\mathbf{e}_{\mathbf{S}}$-Tomo XIII-Buenos Aires, 1935-Broch.-1 vol.-Permuta.

Revista do Archivo Municipal de São Paulo-Vols. XVII, XVIII, XIX e XX -São Paulo, 1935/1936-Brochs,-4 vols.-Permuta.

Revista do Fôro-Vols.XXXI e XXXII —João Pessôa-Parahyba, 1934-Brochs.-2 vols.--Permuta.

Revista do Departamento Nacional da Producção Animal-Vol. I-Rio de Janeiro, 1934-Broch.-1 vol.-Permuta.

Revista do Instituto de Café do Estado de São Paulo-São Paulo, 1935 -Broch.-1 vol.-Permuta.

Revista do Museu Paulista-Vol. XIX -São Paulo, 1935-Broch.-1 vol.Permuta.

Revista dos Tribunaes-Vols. XCVI, XCVII, XCVIII e XCIX-São Paulo, 1935/1936-Brochs. -4 vols.-Permuta.
Revista Forense-Vols. LXIV e LXV Bello Horizonte, 1935-Brochs,-2 vols.-Permuta.

Revista Javeriana-Tomo II-Bogotá, 1934-Broch,-1 vol-Permuta.

Revista Jadiaria-Vol. I-São Paulo, 1935-Broch.-1 vol.-Dermuta

Revista Judiciaria e de Classes-Cruzeiro, 1935-Broch.-1 vol.-Permuta.

Revista Juridica-Orgão Official do Instituto da Ordem dos Advogados de Pernambuco-Vol. IV-Recife, 1934-Broch.-1 vol.-Permuta.

Revista Juridica de Catalunya-Vol. XLI-Barcelona, 1935-Broch.-1 vol. -Permuta.

Revista Universitaria-organo de la Universidad Menor del Cuzco-N. ${ }^{\circ}$ s. 66 e 67-Cuzco-Perú, 1934-Brochs. -2 vols.-Permuta.

Revue de Droit et de CriminologieLouvain, 1935-Broch.-1 vol.-Compra.

Rivadavia Corrêa Meyer-Emprestimos hypothecarios 1934/1935-Caixa Economica do Rio de Janeiro (Relatorio)-Rio de Janeiro, 1935-Broch. -1 vol.-Doação do Dr. Waldemar Ferreira.

Rivista di Diritto Commerciale Industriale e Marittimo-Annos de 1903 a $1910,1912,1913,1915,1916.1919$ a 1924 e 1935-Milano-Brochs.-20 vols.-Doação.

Rivista di Diritto Internazionale-Vol. XIV-Roma, 1935-Broch.-1 vol.Compra.

Rivista di Diritto Penitenziario-Roma, 1935-Broch.-1 vol.-Permuta.

Rivista di Diritto processuale CivilePadova, 1924/1935-Brochs. -24 vols. -Compra.

Rivista Internazionale di Filosofia del Diritto-Roma, 1935-Broch.-1 vol. -Compra.

Rivista Internazionale di Scienze Sociale-Milano, 1935-Broch.-1 vol.Permuta.

Rôle et formation du Bibliothécaire -Paris, 1935-Broch.-1 vol.-Compra. 
Studi Urbinati-Rivista di Scienze Giuridiche-Vols. XII e XIII-Urbino, 1934-Broch._-2 vols._-Permuta.

Temis-Revista scientifica e juridica -Vol. I-Porto Alegre-Rio Grande do Sul, 1934 -Broch.-1 vol.-Permuta.

The Annals of the American Academy of Political and Social Science Vols. 182, 183, 184-Philadelphia, 1935/1936-Brochs.-3 vols.-Compra.

The Glasgow University Calendar 1935/1936-Glasgow, 1935-Encad.1 vol.-Permuta.

Trabajos de Seminario-Tomos V, VI, VI e VIII-Rosario, 1931/1933/1934 -Brochs.-4 vols.-Permuta.

Universidad de la Habana-Publicación bimestral-La Habana-Cuba, 1935Broch.-4 vols.-Permuta.

Wanderley Pinho-Cartas do Imperador D. Pedro II ao Barão de Cotegipe-São Paulo, 1933-Broch.—1 vol.-Comçra.

\section{FILOSOFIA}

Alcides Bezerra-A Philosophia na phase colonial (Conferencia)-Rio de Janeiro, 1935-Broch.-1 vol.Doação do autor.

Arthur Ramos-Freud, Adler, YungEnsaios de psychanalyse orthodoxa e heretica-Rio-Broch.-1 vol.Compra.

Dumas (Georges)-Nouveau Traité de Psychologie-Paris, 1934_Encad.-1 vol.-Compra.

EI regientador diel Mundo solar os Raios de luz-Barcelona, 1934Broch.-1 vol.-Permuta.

Francisco Ribeiro dos Santos-Psychologia e logica-3.. edição-São Paulo, 1936-Broch.-1 vol.-Doação do autor.

Galvão Bueno (C. M.) —Noções de Philosophia-São Paulo, 1877-Encad.1 vol.-Doação.

Mercier (D. J.)-Cours de Philosophie -vols. II e IV-Lovain-Paris, 1923 -Broch._2 vols.-Compra.
Renato Kehl-Typos vulgares (Contribuição á Psychologia pratica)-Rio de Janeiro, 1936-Encad.-1 vol.Doação do autor.

Revue Philosophique de la France et de l'ktranger-Tome CXIX-Paris, 1935-Broch.-1 vol.-Compra.

Ruggles (Arthur Hitler)-Mental Health-Baltimore, 1934-Encad.- vol. --Permuta.

Un drama del espacio-Barcelona, 1932 -Broch.-1 vol.-Permuta.

Un espirito que busca la verdadBarcelona, 1932-Broch.-1 vol.Permuta.

Un espirito que quiere el bien a la humanidad-Dictado por Gabriel Delanne-Barcelona, 1932-Broch.-1 vol.-Doação do Grupo "Amor y Vida"-Barcelona.

Un espiritu que quiere enseñar al hombre de la tierra-Dictado por León Denis-Barcelona, 1932-Broch.-1 vol.-Doação.

Una ciencia desconocida del hombre de la tierra-Barcelona, 1932-Broch. -1 vol.-Permuta.

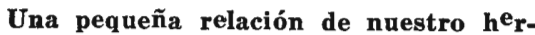
mano Allan Kardec pidiendo misericordia a Dios y al Divino Maestro, para sus hermanos terrenales-Barcelona, 1932-Broch.-1 vol.-Permuta.

\section{RELIGIÃO}

(2)

Berthe (R. P.)-Santo Affonso de Ligorio 1696/1787-São Paulo, 1931Broch.-1 vol.-Doação de Officinas Graphicas Santuario d'Apparecida

Hartmann (Edouard)-La Religion de l'Avenir-Paris, 1881-Encad.-1 vol. -Doação.

Oscar Chagas de Azeredo-Á sombra da Cruz do Redemptor-Apparecida, 1934-Broch.-1 vol.-Doação.

Oscar Chagas de Azeredo-São Clemente Maria Hofbauer-Apparecida. 1928 -Broch.-1 vol.-Doação.

S. Tomás de Aquino-Suma Teológica _Vol I-São Paulo, 1934-Broch.1 vol.-Doação do Prof. Dr. Alexandre Correia. 
XXXII Congresso Eucaristico Internacional-Buenos Aires, 1935-Broch.2 vols.-Permuta.

\section{SOC. DIREITO}

A Acção da Bancada Paulista "Por São Paulo Unido" na Assembléa Constituinte-O programma da "Chapa Unica" e a nova Constituição-São Paulo, 1935-Broch.-4 exempls.-Doação do Dr. Alcantara Machado.

A defesa da instituição da familiaParecer da Camara Corporativa sobre o projecto de lei n. ${ }^{\circ}$ 25Lisboa, 1935-Broch.-1 vol.-Doação de José Gabriel P.nto Coelho.

A. F. Cesarino Junior-O regimen das sociedades anonymas no Brasil, em sua evolução historica-São Paulo, 1935-Broch.—1 vol.-Doação do autor.

A. Pompêo-Porque é que sou integralista-São Paulo, 1935-Broch.1 vol.-Doação do autor.

A Taxa de 3 Shillings no regime do decreto 5.084 de 30 de Junho de 1931-Defesa da Fazenda do Estado-São Paulo, 1936-Broch.-1 vol. -Doação do Dr. Manoel Vieira.

Abelardo Vergueiro Cesar-Lei $n .^{\circ} 160$, de 31 de Dezcmbro de 1935, que altera a Carteira de RetdescontosSão Paulo, 1936-Broch.-1 vol.Doação do autor.

Aben-Attar Netto-Philosophia politica brasileira (Estudos)-São Paulo, 1936-Broch.-1 vol.-Doação do autor.

Actorum Academiae Jurisprudentiae Comparativae-Paris, 1928-Ecad.1 vol.-Compra.

Actos do Municipio de São Paulo do anno de 1935-Actos de n. ${ }^{\circ}$ s. 762 a 990-São Paulo, 1936-Broch.-1 vol. -Permuta.

Affonso Dionysio-Das procuraçõesRio de Jane:ro, 1911-Broch.-1 vol. -Doação.

Alberto J. Robbe-Usura, Moratoria, Reajustamento Economico-São Pau- lo, 1935-Broch.-1 vol.-Doação do autor.

Alberto Rangel-Rumos e perspectivas -São Paulo, 1934-Broch.-1 vol.Compra.

Alcides Bezerra-Aspectos anthropogeographicos da Constituição-Rio de Janeiro, 1935-Broch.-1 vol.Doação do autor.

Alfredo Büchner Lopes da Craz-Aggravo $\mathrm{n} .^{\circ}$ 15.458-Supremo Tribuna] de Justiça do Est. de São PauloSão Paulo, 1928-Broch.-1 vol.Doação de Paulo de Macedo Couto.

Alfredo Büchner Lopes da Cruz-Appelação Civil n. ${ }^{\circ} 20.064$, da Comarca de Santos-São Paulo, 1933Broch.-1 vol -Doação.

Alfredo Büchner Lopes da Cruz-Das denominações necessarias e vulgares como elemento de marca complexa -São Paulo, 1930-Broch.-1 vol.Doação.

Alfredo Büchner Lopes da Cruz e Joaquim Móra de Freitas-A questão da marca " $\mathrm{Ru}$ "-Recurso crime $\mathbf{n} .^{\circ}$ 7.092-São Paulo, 1935-Broch.-1 vol.-Doação de Paulo de Macedo Couto.

Almeida Nogueira-Fiança ás custas São Paulo, 1909-Broch.-1 vol.Doação.

Altavilla (Enrico)-Manuale di Diritto Penale-Napoli. 1934-Encad.-1 vol.-Doação do autor.

Altavilla (Enrico)-Manuale di Procedura Penale-Napoli, 1935-Encad.1 vol.-Doaçâo do autor.

Americo Brazilio Silvado-O extremismo retrógrado integralista e a sâ politica-Rio, Julho de 1935-Broch. -1 vol.-Doação do Dr. Demetrio Lemos.

Anales de Médicine Légale, de Criminologie et de Police ScientifiqueParis, 1935-Broch.-1 vol.-Compra.

Annaes Forenses do Estado de MattoGrosso-Vol. XIII-Cuyabá, 1935Broch.-1 vol.-Permuta.

Ant ${ }^{e}$-projecto de uniformização da contabilidade das Camaras Municipaes do Estado de São Paulo-São Paulo s/d. Broch.-1 vol.-Permuta. 
Antokoletz (Daniel)-Código CivilBuenos Aires, 1931-Broch.-1 vol. - Compra.

Antonio Pinto Cardozo de MelloDesquite-Allegações finaes da autora-São Paula, 1934-Broch.-1 vol. -Doação do Dr. José Carlos de Macedo Soares.

Antonio Sexgio-Educação CuivicaPorto-Broch.-1 vol_-Doação.

Anzilotti (Dionisio) - Curso de Derecho Internacional-Tomo I-Madrid, 1935-Broch.-1 vol--Doação.

Apelt (Ernest Hans)-Die sogenannten Ausnahmenlehre-Breslau, $\quad 1934$ Broch.-1 vol.-Permuta.

Apelt (Ernest Hans)-Die sogenannten in der Teilnahmelehre-Breslau, 1934 -Broch.-1 vol.--Permuta.

Archives de Philosophie du Droit et de Sociologie Juridique-N: $0^{\circ}$ 3-4Paris, 1935-Broch.-1 vol.-Compra.

Airchivo Judiciario-Vol. XXXVI e XXXVII-Rio de Janeiro, 1935/1936 -Brochs.-2 vols.-Permuta.

Archivos da Assistencia a psychopatas de Pernambuco-N. ${ }^{\circ}$ s.1 e 2-Recife, Pernambuco, 1935-Broch.-1 vol.Permuta.

Archivos de Medicina Legal e Identificação-N. ${ }^{\circ}$ 12-Rio de Janeiro, outubro de 1936-Broch.-1 vol.-Permuta.

Ary Machado Guimarães-A evolução do cinematographo-Rio de Janeiro, 1930-Broch.-1 vol.-Doação.

Ascoli (Alfredo)-Trattato delle donazioni-Milano, 1935-Broch.-1 vol.Compra.

Augusto Teixeira de Freitas-Primeiras linhas sobre o processo civilTomo III-Rio de Janeiro, 1879Encad.-1 vol.-Doação.

Azevedo Amaral-o Brasil na crise actual-São Paulo, 1934-Broch.-1 vol.-Compra.

Baethgen (Raul E.)-Noticia general sobre la Abogacia en el UruguayMontevideo, 1935-Broch.-1 vol:Doação do Gremio de Cultura Mauá -Pelotas.
Baldi (Cesare)-Manuale pratico di Diritto Civile-Torino, $1932-2$ vols. -Compra.

Balogh (Elemer)-Actorum Academicae Jurisprudentiae Comparativae-Paris, 1934-Broch -4 vols-Compra.

Bandenhoop (Walter)-Grenzen der deutschen Gebietshoheit bei den Völferrechtlichen und deutschen Sicherheitsanforderungen an die Gersehissahrt-Breslau, 1934-Broch.-1 vol.-Permuta.

Baptista Pereira-Pelo Brasil maiorSão Paulo, 1934-Broch.-1 vol.Compra.

Barão da Penha-Projecto de Regulamento para Prisões Militares organizado pela Commissão de Exame de Legislação do Exercito,Rio de Janeiro, 1877-Broch.-1 vol-Doação do Prof. Dr. Francisco Morato.

Barassi (Lodovico)-Diritto del Lavoro e assicurazioni sociale-Milano -Broch.-2 vols.-Compra.

Barassi (Ludovico)-Il Diritto Reali Milano, 1934-Broch.-1 vol.- Compra.

Barassi (Ludovico)-Instituizic yi di Diritto Privato-Milano, 1933-Broch. -1 vol.-Compra.

Barassi (Ludovico)-Le obligazioni con speciale riguardo di contrati-Milano, 1934-Broch.-1 vol.-Compra.

Barassi (Ludovico)-Teoria della ratifica del contratto annullabile-Milano, 1898-Broch -1 vol.-Doação.

Barbot (Raul)-De las sucesionesMontevideo. 12929-Broch.-2 vols.Compra.

Batardon (Leon) - Tratado pratico de Sociedades Mercantiles-Encad.-1 vol.-Compra.

Baudoin (Louis) - De L'Administration des Successions Ab Intestat en Droit Anglais-Paris, 1928-Broch.-1 vol. - Compra.

Bauer (Arthur)-Les Classes Sociales Paris, 1902-Broch.-1 vol.-Compra.

Bellefonds (Yron Linant)-Des donations en Droit Mulsuman-Paris, 1935-Broch.-1 vol.-Compra. 
Bellèzza (Giuliano)-Technica Mercantile-transporte-dagne-Milano, 1935 -Broch.-1 vol.-Compra.

Bendicente (Francisco C.)-Comisiones de Servicios Publicos MunicipalesRosario, 1935-Broch.-1 vol.-Doação do autor.

Bento de Faria-Decisões da Côrte Suprema-Vol. I-Rio de Janeiro, 1935-Broch.-1 vol.-Compra.

Berg (Roger)-Le Socialisme entre 1'Economie nationale et le Cosmopolitisme-Paris, 1935-Broch -1 vol. -Compra.

Berthélemy (H.)-Traité Elementaire de Droit Administratif-Paris, 1933 -Broch.-1 vol.-Compra.

Bethman (Werner)-Die Kollision von Rechtsgeschäften bei gesetzlicher und gewillkürter StellvertretungBreslau, 1934-Broch.-1 vol.-Permuta.

Bittner (Hans)-Der Prozefzevegleich im Privatklageverfahren-Breslau, 1934-Broch.-1 vol.-Doação.

Blume (Runz)-Das sehlefische Runstchandwerbe-Breslau, 1933-Broch. -1 vol.-Doação.

Bonfante (Pietro)-Seritti Giuridici Varii-Torino. 1926-Broch.-3 vols. Compra.

Bonnecase (Julien)-Traité théorique et pratique de Droit Civil-Paris, 1926/1930-Broch.-3 vols.-Compra.

Bonnet (Victor)-Le Crédit et les Banques d'Emission-Paris, 1875Brach.-1 vol.-Doação do Prof. Dr. Francisco Morato.

Bouza (Luis Alberto)-El homicidio por piedad y el nuevo Código $\mathbf{P e}$ nal-Montevideo, 1935-Broch.-1 vol.-Compra.

Brasil e Japão-duas civilizações que se contemplam-São Paulo, 1934Broch.-1 vol.-Doação do Consulado do Japão.

Brissaud (J.)-Manuel d'Histoire du Droit Privé--Paris, 1935-Broch.-1 vol.-Compra.

Branner (Heinrich)-Historia del Derecho Germanico-Barcelona, 1936Encad.-1 vol.-Compra.
Brunschwik (Lisa)-La responsabilité envers les tiers dans navegation aèrienne-Paris, 1935-Broch.-1 vol. - Compra.

Campos Salles-Da propaganda á Presidencia-São Paulo, 1908-Broch.1 vol.-Doação.

Candido Baptista de Oliveira-Systema Financial do Brasil-S. Petersburgo, 1842-Encad.-1 vol.-Doação do Prof. Dr. Francisco Morato.

Cantalupo-A Abyssinia preparava a guerra á Italia-Rio de Janeiro, 1935-Broch.-1 vol.-Doação do Regio Consolato Generale d'Italia.

Capitant (Henri) - La Thèse de Doctorat en Droit-Paris, 1935-Broch.1 vol.-Compra.

Carlos Antonio Cordeiro e Manuel Godofredo de Alencastro AutranConsultor orphanologico acerca de todas as acções seguidas no Juizo de Orphãos-Rio de Janeiro, 1880Encad.-1 vol.-Doação.

Carlos Fernandes-De Jure Vitae Necisque-Rio de Janeiro, 1933-Broch. -1 vol.-Doação do autor.

Carlos Maximiliano-Commentários á Constituição Brasileira-Porto Alegre, 1929-Encad.-1 vol.-Compra.

Carlos Xavier Pares Barretto-Processualistica Criminal-Critica ao Projecto do Codigo de Processo Penal Brasileiro-Victoria, 1936-Broch.1 vol.-Doação do autor.

Carneiro (Manuel Borges)-Direito Civil de Portugal-Lisboa, 1858Encad.-4 vols.--Doação.

Carnelutti (Francesco)-Lezioni di Diritto Processuale Civile-Padova, 1933-Broch.-1 vol.-Compra.

Carnelutti (Francesco)-Lezioni di Diritto Processuale Civile-_Processo di Esecuzione-_Padova, 1932/1933Encad.-3 vols.-Compra.

Carnelutti (Francesco)-Studi di Diritto Processuale-Padova, 1928/1935 -Encads.-2 vols.-Compra.

Castelar (Emilio)-La fómula del progreso-Madrid, 1870-Encad.--1 vol.-Doação. 
Celisa Ribeiro de Arruda-Como estudar com efficiencia-São Paulo, Broch.-1 vol.-Doação da autora.

Celso Vieira-Socialisação NacionalRio, 1933-Broch.-1 vol.-Doação do autor.

Clovis Moreira Spinola-Projecto de um Codigo de "Ethica Profissional" -Bahia, 1922-Broch.-1 vol.-Doação.

Cocurullo (Baldassare) - I moventi a delinquere-Napoli, 1930 -Broch.-

1 vol.-Compra.

Code des Obligations de la République de Pologne-Paris, 1935-Broch. -1 vol.-Doação do Dr. Aleksander W. Matuszewski.

Codigo Civile del Regno d'Italia-Torino, 1901-Encad.-1 vol.-Doação.

Codice di Commercio del Regno d'Italia-Torino, 1901-Encad.-1 vol.Doação.

Codice di Procedura, Penali per il Regno d'Italia-Torino, 1915-Encad. -1 vol.-Doação.

Código Civil Español-Madrid-Encad. -1 vol.-Doação.

Codigo do Processo Civil e Commercial do Estado de São Paulo-São Paula, 1930-Broch.-11 exemplares -Doação da Secretaria da Justiça e Segurança Publica.

Codigo Tributario do Estado de Minas Geraes-Minas Geraes, 1936-1 vol.-Permuta.

Coelho (José Gabriel Pinto)-A successão dos parentes illegitimos e a reforma do Codigo Civil-Lisboa, 1935-Broch.-1 vol.-Doação do autor.

Collecção de leis do Brasil-Rio de Janeiro, 1868-1869-Encad.-3 vols. -Doação.

Collecção de leis e decretos do Estado de São Paulo-1933-Tomo XLV1.0 semestre-São Paulo, 1935Broch.-1 vol.-Doação.

Collette (Jean)-Les principes de droit des gens dans la jurisprudence de Court Permanente de Justice Internationale-Paris, 1932-Broch.-1 vol.-Permutado.
Columbia Law Review-Vol, XXXVNew-York, 1935-Broch.-1 vol.Permuta.

Commissão de Finanças e Orçamento - Camara dos Deputados-Rio de Janeiro, 1935-Broch.-1 vol.-Doação.

Congrés Juridic Català-Barcelona, 1936-Broch.-1 vol.-Permuta.

Congresso de 1 a Mutualidad-Museo Social Argentino-Buenos Aires, 1917 -Broch.-1 vol.-Doação da Bibl. Nacional de Buenos Aires.

Conselho de Contribuintes-Ministerio da Fazenda-Diario OfficialRio de Janeiro, 1935/1936-Brochs. -2 vols.-Compra.

Consolidação das Leis Civis-Rio de Janeiro, 1876-Encad.-1 vol.-Doação.

Constituição do Estado de Matto Grosso-Cuyabá, 1935-Broch.-1 vol. -Doação do Dr. Oscar Correia Pina.

Constituição do Estado de São Paulo -São Paulo, 1935-Broch.—1 vol.Compra.

Constituição do Estado do Espirito Santo de 11 de Agosto de 1935Victoria, 1935-Broch.-1 vol.-Doação da Bibliotheca da Reitoria da Universidade de São Paulo.

Constituição Politica do Estado do Pará-Pará-Belém, 1935-Broch.-1 vol.-Doação do Dr. José Carlos de Macedo Soares.

Corbu (Adrien C.)-Essai sur la notion de Règle de Droit en Droit International-Paris, 1935-Broch.-1 vol.-Compra.

Cornélissen (Christian)-Les générations nouvelles-Paris, 1935-Broch. -1 vol.-Compra.

Cossa (Emilio)-Conflitti e alleanze di capitale e lavoro-Milano, 1903Broch.-1 vol.-Doação.

Coste-Floret (Alfred)-La Nature Juridique du Mariage-Paris, 1935Broch.-1 vol.-Compra.

Cours de Droit Administratif-Paris, 1933-Broch.-2 vols.-Compra.

Credito movel-Decreto $n .^{\circ}$ 169-A de 
19 de Janeiro de 1890 -Rio de Janeiro, 1891-Encad.-1 vol.-Doação.

Crew (Albert)-Ecinomia-Barcelona, 1934-Encad.-1 vol.-Compra.

D N C-Revista do Departamento Nacional do Cofé-Vol. V-Rio de Janeiro, 1935-Broch.-1 vol.-Permuta.

Dareste (P.)-Les Constitutions Modernes-Paris. 1928-1933-Broch.-6 vols.-Compra.

De Page (Henri)--A propos du gouvernement des juges l'équité en face du droit-Bruxellas-Paris, 1931Broch.-1 vol.-Compra.

Decisões do Superior Tribunal do Estado do Rio Grande do Sul, proferidas durante o anno de 1932-Porto Alegre, 1934 -Broch.-1 vol.-Permuta.

Decreto n. ${ }^{\circ} \mathbf{7 3 9 2}$ de 25 de Setembro de 1935-Regulamento da Faculdade de Pharmacia e Odontologia da Universidade de São Paulo-São Paulo, 1936-Broch.-1 vol.-Doação da Secretaria de Educação e Saúde Publica.

Decretos e resoluções do Governo Provisorio de São Paulo de 18 de Novembro de 1889 a 30 de Junho de 1891-São Paulo, 1897-Broch.—2 vols.-Doação.

Deschamps (Jacques)-L'aggravation du risque en cours d'assuranceParis, 1933-Broch.-1 vol-Compra.

Ditt (Bruno)-Das StrasklagerechtBreslau, 1934-1 vol.-Doação.

Dobranich (Horacio H.)-Dictamenes Fiscales-Buenos Aires, 1934-Broch. -1 vol.-Permuta.

Döring (Werner)-Das Prioritätsrecht des Art. 4-Breslau, 1934-Broch1 vol.-Permuta.

Dunedin (Viscount)-The Divergencies of English and Scottish Law-Glasgow, 1935-Broch.-1 vol.-Permuta.

Eckert (Max)-Das Recht der polizeilichen Verfügung nach dem Preuzischen Polizeiverwarltungsgesetz von 1 Juni 1931-Breslau-Broch.1 vol.Doação.
Edital de concorrencia publica para as obras de melhoramentos do Porto de São Sebastião-Sāo Paulo, 1935Broch.-1 vol.-Doação da Secretaria da Viação e Obras Publicas do Estado de São Paulo.

Eduardo Santos-Discurso pronunciado el 21 de febrero de 1933-ante la Sociedad de las Naciones, con motivo de la acusación formulada contra el gobierno del Perú por la invasión peruana a territorio colombiano-Broch.1 val.-Doação

El conflicto de Leticia-Madrid, 1933 -Broch.-1 vol_-Doação da Legacion de Colombia en España.

Em memoria de Antonio de Alcantara -1 vol.-Doação do Dr. Alcantara Machado.

Espinas (Alfred)-Des Sociétés Animales-Paris, 1935-Broch.-1 vol. -Compra.

Essad Bey-A luta pelo petroleo-São Paulo, 1935-Broch.-1 vol.-Doação da Livraria Universal.

Estudios Internacionales-Publicaciones del Grupo Español de la União ide Friburgo-Madrid. 1935-Broch.

-Doação do Prof. Dr. Alfredo Mendizábal Villalva.

Evaristo de Moraes-Carceres e fogueiras da inquisição-Rio de Janetiro-Broch.-1 vol--Compra.

Fabiani (Andrea)-Del falso ideologico nel delitto documentale-Napoli, 1933-Broch.-1 vol.-Compra.

Ferien-Curse in Deutschland-Deutschen Akademischen Austanschdienst E. V.-Berlim, 1936-Broch.-1 vol. -Permuta.

Fernando Azevedo-Principios de sociologia-São Paulo, 1935-Broch.1 vol.-Compra.

Ferrara (Francesco)-Della simulazione dei negozi giuridici-Milano, 1905 -Broch.-1 vol.--Doação.

Ferrari (Francesco Antonio)-L'Usura nel Diritto, nella Storia, nell'Arto -Napoli, 1928-Broch.-1 vol.Compra. 
Finzi (Marcello)-Il Museo Criminale di Roma-Citta di Castello, 1932Broch.-1 vol.-Doação do autor.

Finzi (Marcello)-Prefazione e appendice alla ristampa del libro di Domenico Giurati: Gli errori giudiziari-Bologna, Broch.-1 vol.-Doação do autor.

Finzi (Marcello)_"Scopo" "fine" "intenta" "intenzione" "motivo" nel codice penale italiano-Napoli, 1932 -Broch.-1 vol.-Doação do autor.

Finzi (Marcello)-Silvio Perozzi e le sue lezioni di diritto penale-Città di Castello, 1935-Broch.-1 vol.Doação do autor.

Fixier (Georges)-Le délit d'émmission de chèque sans provisionNancy, 1934 -Broch.-1 vol.-Compra.

Flaminio Favero-A pericia medica em caso de defloramento-São Paulo, Broch.-1 vol.-Doação do autor.

Flaminio Favero e Elisa Norah-Da applicação da colorimetria para a dosagem do oleo essencial de sabina-São Paulo-Broch.-1 vol.Doação do autor.

Flaminio Favero e Manuel PereiraA cinematographia na documentacão de damnos deformantes perceptiveis em movimento (Sobre um caso de hemiplegia de origem subcortical)-São Paulo, 1935-1 vol.Doação dos autores.

Flöb (Walter)-Goldknappheit und Goldverteilung-B r e s l a u, 1934 Broch.-1 vol.-Doação.

Florian (Eugenio)-Principi di Diritto Processuale Penale-Torino, 1932 -Broch.-1 vol.-Compra.

Folliet (Joseph)-Le droit de colonisation-Etude de morale sociale et internationale-Paris, Broch.-1 vol. -Compra.

Fontanilles (J. Conangiola)-La naturaleza de las casas-Ensayo para un estudio de geografia PoliticaLa Habana, 1935--Broch.-1 vol.Doação do autor.

Förster (Edgar)-Der Einfluk-Breslau, 1934-Broch.-1 vol.-Doação da Universidade de Breslau.
Fraenfei (Kurt)-Niessbranch und Znvangsverwaltung-Breslau, 1934Broch.-1 vol.-Doação.

Fragali (Michele)--Principi di diritto aeronautico-Padova, 1930-Broch. 1 vol.-Compra.

Franceschelli (Remo)-Il “Trust" nel Diritto Inglese-Padova, 1935Broch -1 vol.-Compra.

Francisco de Paula Rodrigues Alves -Plano de reforma judiciaria submettido ao Congresso do Estado de São Paulo-São Paulo, 1912-Broch. -1 vol.-Doação.

Fundo da questão da MandchuriaNew York s/d.-Broch.-1 vol.-Doação.

Fuzier-Herman-Code Civil annotéParis, 1935-1936-Broch.-2 vols.Compra.

Garçon (E.)-Code Pénal AnnotéParis, 1901-1930-Broch.-3 vols.Permutado.

Garnier (M. le Marquis)-Histoire de la Monnaie-Paris, 1819-Broch.-2 vols.-Doação.

Genil-Perrin-Psychanalyse e Criminologia-Rio, 1936-Broch.-1 vol. -Compra.

Gilka (Gerhart)-Ist § 46 St G B bei allen straf baren Vorbereitungshandlugen des Strafgesetzbuchs per argumentum a maiori ad minus anwendbar?-Berlim-Broch.-1 vol. -Permuta.

Girardin (Emile)-L'Impot-Paris, 1852 -Encad.-1 vol.-Doação.

Godofredo de Faria-Paiz aviltadoRio de Janeiro, 1935-Broch.-1 vol. -Doação do autor.

Goerke (Herbert)-Keynes Geldlehre Darstelung und Kritik-Breslau, 1934-Broch.-1 vol.-Doação.

Goldschmidt (James)-Derecho Procesual Civil-Madrid, 1936-Encad. -1 vol.-Compra.

Goldschmidt (Roberto)-Recenti tendenze nel diritto della società aunnima-Firenze, Broch.-1 vol.--Permuta. 
Golling (Banl)-Die Problematik der Landarbeiterorganisation - Breslan, 1934-Broch.-1 vol.-Permuta.

Goodnow (Frank Johnson)-The Colver Lectures 1916. The American Conception of Liberty and The American Conception of Government-Providence, 1916-Broch.-1 vol.-Permuta.

Gouveia (Jaime Augusto Cardoso de) -Da responsabilidade contractualLisboa, 1933-Broch.-1 vol.-Compra.

Guelfi (G. Filomusi)-Trattato di Medicina Legale-Napoli, 1908-Encad.-1 vol.-Permutado.

Habicht (Max)-Post-War Treaties for The Pacific Settlement Disputes -Cambridge. 1931-Encad.-1 vol. - Permuta.

Hanke (Hellemuth)-Frankreichs Stelung zur Meistbegünstigungsklansel -Breslau, 1934-Broch.-1 vol.Doação.

Hardenberg (Albrecht) Graf von)Das Recht der freien Meinungsäusserung in Art. 118 Abs. I der Reichsverfassung und die Schadensersatzansprüche ans seiner Verletzung-Breslau, 1933-Broch.-1 vol.-Permuta.

Harris (S. E.)-Monetary Problems of the British Empire-New York, 1931-Encad.-1 vo!--Permuta.

Hartmann (Hans-Georg)-Die Erfüllungsverweigerung mit besonderer Berücksichtigung der positiven Vertragsiverletzungen-Breslau, 1934 -Broch.-1 vol.-Doação.

Harvard Law Review-Vol. XLVIIIMass, U. S. A., 1934-Broch.-1 vol. -Permuta.

Heinsheimer (Karl)-Derecho Mercantil-Barcelona, 1933-Broch-1 vol.-Compra.

Heitor Bracet-A estimativa da população do Brasil-Rio de Janeiro, 1935-Broch.-1 vol.-Doação do Dr. Waldemar Ferreira.

Henri Mazeaud et León MazeaudTraité théorique et pratique de la responsabilité civile delictuelle et contractuelle-Paris, 1934-Broch.1 vol.-Compra.

Henrique Coelho-O Poder Legislativo no Direito Publico Brasileiro-São Paulo, 1905-Broch.-1 vol.-Doação.

Hernandez (Eusebio Adolfo)-Tratado de Derecho Penal-Habana, 1918 -Broch.-1 vol.-Permutado.

Hershey (Amos S.)-The Essentials of International Public Law-New York, 1919-Encad.-1 vol.-Permutado.

Herzka (Franz)-Die Legitimations übertragung-Breslau, 1934 -Broch. -1 vol.--Doação.

Hildebrando Accioly-O reconhecimento do Brasil pelos Estados Unidos da America-São Paulo, 1936-Broch.-1 vol.-Compra.

Höhl (Joachim)-Das Recht ans dem Antrage unter besonderer Berücksichtigung der Grundstücksosserte -Breslau, 1934-Broch.-1 vol.Permuta.

Horoy-Des Rapports du SacerdoseParic, 1883-1884-Broch.-2 vols.Doação do Prof. Dr. Francisco Morato.

Hulot-La Clef des Lois Romaines, on Dictionnaire-Tome I-Metz, 1809Encad.-1 vol.-Doação.

Halot-Les Cinquante Livres du Digeste ou des Pandectes-Paris, 18031805-Encad.-5 vols.-Doação.

Hyppolito de Camargo-Menores e interdictos-Estudo pratico sobre tutelas e curatelas-São Paulo. 1891 -Encad.-1 vol.--Doação.

Industrias y Finanzas-Revista Técnica de Bancos y Seguros-Buenos Aires, 1935-Broch.-1 vol.-Permuta.

International Conciliation-Documents for the year 1935-New York City, 1935-Broch.-1 vol.-Permuta.

Ishaq (Halil Ibn)-Il "Muhtasar" o Sommario del Diritto MalechitaMilano, $1919-$ Broch.-1 vol.-Compra. 
J. do Amaral Gurgel-Desquite (Theoria e Pratica)-São Paulo, 1936Encad.-1 vol.-Compra.

J. M. de Azevedo Marques-Acções de despejo e alugueres de prediosSão Paulo, 1918-Encad.-1 vol.Doação do autor.

J. M. de Carvalho Santos-Codigo Civil Brasileiro interpretado-Vols. XI e XII-Rio de Janeiro, 1936Brochs.-2 vols.-Compra.

J. Peçanha de Figueiredo-Terras devolutas-São Paulo, 1936-Broch. -1 vol.-Compra.

J. X. Carvalho de Mendonça-Tratado de Direito Commercial BrasileiroRio de Janeiro, 1933-Encad.-11 vols.-Compra.

Jacks (L. P.) -Ethical Factores of The Present Crisis-Baltimore, 1934 -Encad.-1 vol.-Permuta.

Jacobsohn (Geert)-Die Wirkungen der Diskontpolitik auf die zahlungsbilanz-Breslau, 1934 -Broch.-1 vol.-Permuta.

Jeager (Nicola)-La Riconvenzione nel Processo Civile-Padova, 1930Encad.-1 vol.-Permutado.

Jaffin (George H.)-Federal Procedural Revision-New York, 1935Broch.-1 vol-Doação do autor.

James Ferraz Alvim-Estudo NeuroPsychiatricos-São Paulo, 1931Broch.-1 vol.-Doação.

João Arruda-Advogados em inventarios a partilhas-O Codigo do Processo em elaboração-São Paulo, 1936-Broch.-1 vol.-Doação do autor.

João Arruda-Do casamento-São Paulo, 1911-Broch.-1 vol.-Doação do Dr. A. Viriato Pereira.

João Gonçalves Dente-Embargos n.o 19.747-Camara Civil do Tribunal de Justiça do Estado de São Paulo -São Paulo, 1921-Broch.-1 vol.Doação.

João Lyra-Cifras e Notas (Economia e finanças do Brasil)-Rio de Janeiro, 1925-Broch.-1 vol.-Permuta.
João Pinto da Costa Leite- Noções elementares de Economia Politica -Coimbra, 1934-Broch.-1 vol.Compra.

Joaquim de Oliveira Machado-O Habeas-Corpus no Brasil-Rio de Janeiro, 1878-Encad.--1 vol.--Doação.

José de Moraes Leme-O problema venereo (estudo hygienico-social)São Paulo, 1926-Broch.-1 vol.Doação do autor.

José Prospero Jehovah da Silva Caroatá- 0 Vademecum forense-Rio de Janeiro, 1881-Encad.-1 vol.Doação.

Josseau (J. B-)-Le crédit foncier de France-Paris, 1860-Broch.-1 vol. -Doação do Prof. Dr. Francisco Morato.

Journal du Droit International-Vol. LXII-Paris, 1935-Broch.-1 vol.Permuta.

Julgados e Decisões do Superior Tribunal de Justiça do Estado do Amazonas 1933 (1.0 semestre)Manáos, 1934-Broch.-1 vol.Permuta.

Jurisprudencia-Diario da JustiçaRio de Janeiro, 1935, 1936-Brochs. -3 vols.-Vols. XII, XIII e XIVCompra.

Jurisprudencia administrativa-Leyes de amparo $y$ accidentes del trabajo -Buenos Aires, 1934-1935-Broch. -2 vols.-Doação do Ministerio del Interior-Buenos Aires.

Knothe (Heino Arnold)-Das Problem des Betriebsrisikos-Breslau, 1934Broch.-1 vol.-Permuta.

Kreller (Hans)-Zur Geschichte Der Exceptio Non Numeratae Pecuniae -Palermo, 1932-Broch.-1 vol.Doação do autor.

La Ginstizia Penale-Vol. XLI-Roma, 1935-Brochs. -4 vols.-Permuta.

La Vie Intellectuelle-Tome XXXIXJuvisy-Seine-et-Oise, 1935-Brach.1 vol.-Permuta.

Lachance (R. P. Louis)-Le concept de droit selon Aristote et S. Thomas 
-Paris, 1933-Broch.-1 vol,-Compra.

Las Islas Malvinas-Buenos Aires, 1936-Broch.-1 vol.-Permuta.

L'Économie Dirigée-Paris, 1934Broch.-1 vol.-Doação de L'Ecole Libre de Sciences Politiques-Poris.

L'Esprit International-The International Mind-Paris, 1935-Broch.1 vol.--Permuta.

Le Fur (Louis)-Précis de Droit International Public-Paris, 1933Broch.-1 vol.-Permutado.

Le Play (M. F.)-La Constitution de l'Anglaterre-Paris, 1875-Broch.-2 vols.-Doação do Prof. Dr. Francisco Morato.

Le Play (M. F.) - La Réforme Sociale en France-Paris, 1874-Broch-3 vols.-Doação do Prof. Dr. Francisco Morato.

Le Play (M. F.)-L'Organisation de la famille-Paris, 1875-Broch.2 vols.-Doação do Prof. Dr. Francisco Morato.

Le Play (M. F.)-L'Organisation du Travail-Paris, 1871--Broch.-3 vols. -Doação do Prof Dr. Francisco Morato.

Leis, decretos e contractos relativos ás concessões vigentes de estradas de ferro, outorgadas pelo Governo de São Paulo, 1869-1913-São Paulo, 1914-Broch.-1 vol.-Doação.

Leis e resoluções decretadjas pelo Congresso Legislativo do Estado de São Paulo em 1891-São Paulo, 1891 -Broch.-1 vol.-Doação.

Ley 26 de 1932 sobre immigracion, decreto $n 0^{\circ} 3$ de 1933 que la reglamenta, $y$ lei 46 de 1934-Panamá, 1936-Broch.-1 vol.-Doação.

Lins e Silva-Projecto 67 (Dispõe sobre verificação de obitos, necroscopias e visceratonias)-Ássembléa Legislativa do Estado de Pernambuco-Recife, 1936-Broch.-1 vol. -Doação do autor.

Liscano (Tomas)-Sobre la influencia del Codigo Napolionico en la Legislacion Civil Venezolano-Cara- cas, 1935-Broch.-1 vol.-Doação do autor.

Lobão (Manoel de Almeida e Souza)Collecção de dissertações juridicas e praticas-Lisboa, 1826-Encad.-1 vol.-Doação do Dr. Francisco Loffredo.

Lobão (Manoel de Almeida e Souza de)-Tratado das Acções Summarias e Summarissimas-Tomo IILisboa, 1860-Encad.-1 vol.-Doação.

Loreto Filho-A disciplina do Direito Internacional Privado; sua bibliographia e seu estudo no Brasil (Conferencia)-Recife, 1935-Broch. -1 vol.-Doação do autor.

Lovell (Reginald Ivan)-The Struggle for South Africa 1875-1899 (A Stu$\mathrm{dy}$ in Economie Imperialism)-New York, 1934-Encad-1 vol.-Permuta.

Luiz M. Correia-Razões e Pareceres -Fortaleza, 1918-Broch.-1 vol.Doação.

Lust (Rudolf)-Die Stellung des Leiters der Gemeinde nach dem Preussischen Gemeindeverfassungsgesetz vom 15-Breslau, 1934Broch.-1 vol.-Dermuta.

M. F. P.-Formulario dos trabalhos das Juntas Parochiaes e Municipaes -R:jo de Janeiro, 1876-Encad.-1 vol.-Doação.

Mac-Pherson (M. J. Gornes)-La Polit ca Monetaria de Venezuela-Caracas, 1934-Broch.-1 vol.-Doação.

Maetschte (Karl)-Dars der Reichpräfidont nach Nrtifel 48, Ubfah 2. Cak 2, Mafsnahmen tressen, die Widerspruch zu der Reichsberfassung stehen ?-Breslau, 1934-Broch. -1 vol.-Doação.

Manoel Godofredo d'Alencastro Aatran-Constituição da Republica dos Estados Unidos do Brasil-Rio de Janeiro, 1892-Encad.-1 vol.Doação.

Manoel Pacheco Prates-Pontos de Direito Civil-São Paulo, 1924 Broch.-1 vol.-Doação. 
Manual Parlamentar-Regimento Interno da Camara dos DeputadosRio de Janeiro, 1883-Encad.-1 vol.-Doação.

Manzini (Vincenza)-Diritto Penale Militare-Padova, 1932-Broch.-1 vol.-Compra.

Manzini (Vincenzo)-Trattato di Diritto Processuale Italiano secondo il nuovo Codice-Torino, 1931-1932 -Encad.-4 vols.-Compra.

Marcadé (V.)-Explication Théorique e Pratique du Códe Napoléon-Paris, 1866-1868-Encad.-12 vols.Doação do Dr. Viriato Pereíra.

Mario de Andrade Ramos-Discurso e projectos (Julho 1934-Abril 1935)Rio de Janeiro, 1935-Broch.-1 vol.-Doação.

Maurach (Bruno)-Die völkrrechtlichen Grundsätze in der sovetrussischen Aukenhandelspolitik-Breslau, 1933-Broch.-1 vol.-Doação.

Meinow (Franz)-Die volkswirtschaftliche Bedentung der Gruppenversicherung-Breslau-Broch.-1 vol. -Permuta.

Memorial apresentado á Camara dos Senhores Deputados pelos docentes militares reformados--Rio, 1935Broch.-1 val.-Doação.

Mercier (J.)-Cours de PhilosophieLovain-Paris, 1935-Broch.-1 vol. -Compra.

Meyer (Hildegard)-Beiträge zu der Lehre von der nichtigen offenen Handelsgesellschaft unter besonderer Berücksichtigung der Sociétẻ de fait des französischen RechtesBreslau, 1933-Broch.--1 vol.-Doação.

Miró (Amadea Hurtado i)-El pensamento juridic Catalá-Barcelona, 1935-Broch.-1 vol.-Permuta.

Montella (R. Gay de)-Código de Comercio Español comentado-Barcelona, s/d.-Encad.-7 vols.-Compra.

Moskowa (M. de la)-Du PapierMonnaie et de la démonétisation des especes-Paris, 1842-Encad.-
1 vol.-Doação do Prof. Dr. Francisco Morato.

Münzer (Helmut)-Die Anwendung von Rechtsgrundsätzen des Privatrechts auf offentliche Recht in der preuzischen Verwaltungspraxis -Breslau, 1934-Broch.-1 vol.Doação.

Navarrini (Umberto)-Trattato di Diritto Fallimentare secondo la nuova legislazione-Bologna, 1934-1935 -Broch.-2 vols.-Compra.

Nencioni (Giovanni)-L'Intervento Voluntario Litisconsorziale nel Processo Civile-Padova, 1935-Broch.Compra.

Neumann (Hans(-Das Beweiszeichen im Lichte des "Um tlichem Strasgesekentwurses" von 1927-Breslau, 1933-Broch.-1 vol.-Permuta.

Nina Rodrigues- $O$ alienado no direito civil brasileiro-Rio, 1933Broch.-1 vol.-Compra.

Nina Rodrigues-Os africanos no Brasil-São Paulo, 1933-Broch.-1 vol.-Compra.

Nitschke (Willibald-Heinz)-Die Ergebnisse der wirtschaftlichen und der formalen theorien bein beim zueignungsbegriff--Breslau, 1934 Broch.-1 vol.-Doação.

Noé Azevedo-A questão do "Hospital Syrio" com um parecer do Dr. Plinio Barreto-São Paulo, 1936Broch.-1 vol.-Doação do autor.

Nova tributação do imposto de consumo (Decreto 22.262 de 28 de Dezembro de 1932, alterado pelo de n. 22.487, de 22 de Fevereiro de 1933)-Rio de Janeiro, 1933-Broch. -1 vol.-Doação da Bibl. do Ministerio da Fazenda.

Nuñez (Eduardo Raphael Nañez y) Codigo Civil-Tomos III, IV e VHabana, 1935-Brochs. -3 vols.Compra.

o penhor segundo a legislação civil e commercial-Rio de Janeiro, 1886 -Encad.-1 vol.-Doação.

o plano de um Governo e as razões de uma opposição-Maranhão, 1935 -Broch.-1 vol.-Doação da Im- 
prensa Official do Estado do Maranhão.

O substitutivo aos projectos de organização do Conselho Nacional de Educação-Rio de Janeiro, 1935Broch.-1 vol.-Doação.

Olbrich (Hans)-Arglist und Rechtskraft-Breslau, $1934-B$ roch.-1 vol. -Permuta.

Oliveira Vianna-Raça e assimilação —São Paulo, 1934-Broch.—1 vol.Compra.

Ordenações e Leis do Rẹino de Portugal-Tomo II-Coimbra, 1865Encad.-1 vol.-Doação.

Ordonnance du Roi da 31 Mai 1838, portant Règlement Général sur la Comptabilité Publique-Paris, 1838 -Encad.-1 vol.-Doação do Prof. Dr. Francisco Morato.

Orlando Gomes-Innovação da Constituição (direitos sociaes, representação profissional, justiça eleitoral) -Bahia, 1933-Broch.-1 vol.-Doacão do autor.

Oscar Tenorio-O problema immigratorio e a Constituição de 1934-Rio de Janeiro, 1935-Broch.-1 vol.Doação.

Pacchioni (Giovanni)-Corso di Diritto Civile-Delle leggi in generale $\longrightarrow$ oTrino, 1933-Broch-1 vol.Compra.

Pacifici-Mazzoni-Instituzioni di Diritto Civile Italiano-Firenze, 18801894 -Encad.-7 vols.-Doação.

Padilla (Justa Roqué de)-La mujer y la moral social (Conferencia) Buenos Aires. 1927-Broch.-1 vol. -Permuta.

Paetzuld (Helmut)-Das Voltsbegeren über das "Freiheits-Gesetz" (1929) als-Duelle staatsrechtlicher Problemstellung-Berlim-Broch.-1 vol. -Permuta

Page (Henri de)-Traité Elémentaire de Droit Civil Belge-Bruxellas, 1933-1934_Broch-_2 vols.-Compra.

Pagot (E.)-Etude sur le Contrat d'Assurance contre les accidentsParis, 1896-Broch.-1 vol.-Doação.
Palma Carlos-Os novos aspectos do Direito Penal-Lisboa, 1934-Broch. -1 vol.-Compra.

Paraná Judiciario-Vol. XXII-Curityba, 1935-Broch.-1 vol.-Permuta.

Pardessus (J. M.)-Cours de Droit Commercial-Tomos III e VI-Paris, 1841-Ecad.-2 vols.-Doação.

Pareceres-Camara dos Deputados-Río de Janeiro, 1935-Broch.-1 vol.Doação.

Paulo Alberto-A Guerra-Bahia, 1935 -Broch.-1 vol.-Doação do autor.

Paulo Cesar Machado-Pela justiçaRio de Janeiro, 1935-Bróch.-1 vol. -Doação.

Paulo Ferreira de Souza-Legislação Florestal-Leis Florestaes dos Estados-Segunda Parte-Rio de Janeiro, 1935-Broch.-1 vol.-Permuta.

Peco (José)-La Reforma Penal en el Senado de 1933-Buenos Aires, 1936 -Broch.-1 vol.-Doação do autor.

Pedro Calmon-Espirito da Sociedade Colonial-São, Paulo, 1935-Broch.1 vol.-Compra.

Perfilze (Hans-Günter) - Die Begünstigung durch den Verteidiger-Breslau, 1934-Broch.-1 vol,-Permuta.

Philippart (Léon)-Cours pratique de Droit Commercial et industrielParis, 1935-Broch.-1 vol.-Compra.

Pietro (Marcial Martinez)-La actitud social frente al delito-SantiagoChile, 1931-Broch.-1 vol.-Compra.

Pohl (Norbert)-Schadensersatz bei Verletzung des $\S 909$ BGB-Breslau, 1934-Broch.-1 vol.-Doação.

Political Science Quarterly-Vol.L_. New York, 1935-Broch.-1 vol.Compra.

Por uma só Bandeira! -Campanha Civica do Centro Carioca-Rio de Janeiro, 1934-Broch.-1 vol.-Doação do Dr. José Carlos de Macedo Soares.

Pouget (M. Louis)-Guide du Créancier Hypothécaire-Paris, 1866-Broch.-1 vol.-Doação. 
Préau (Gaston)-Lé contrat de travail -Paris, 1934-Encad.-1 vol.-Compra.

Presutti (Enrico)-Instituzioni di Diritto Amnistrativo Italiano-Messina, 1931/1934-Broch.-3 vols.-Compra.

Primeira Conferencia Nacional sobre analfabetismo-Buenos Aires, 1935 -Broch-1 vol.-Doação da Biblioteca Nacional de Buenos Aires

Proceedings of the Academy of Political Science-Vol. XVI--Broch.1 vol.-Permuta.

Projectos-Senado Federal e Camara dos Deputados-Rio de Janeiro, 1935 -Broch.—2 vols.-Doação do Dr. Waldemar Ferreira.

Pufendorf (Samuel)-De Jure Naturae et Gentium Libri Octo-London, 1934 -Encad.-2 vols.-Permuta.

Quinteros (José S.)-Derecho Admistrativo-La Paz-Bolivia, 1920Broch.-1 vol.--Permuta.

R. Oliva-Desapropriação-II parteSão Paulo, 1936-Broch.-1 vol.Doação do autor.

R. Oliva-Testamento Publico-Quando póde ser assignado por terceiro, sabendo o testador escrever-São Paulo. 1935-Broch.-1 vol.-Doação do autor.

Ranelletti (Oreste)-Istituzioni di Diritto Pubblico-Padova, 1935-Encad. -1 vol.-Compra.

Razões da Fazenda do Estado de São Paulo numa aç̧ão de restituição do Tres Shillings-São Paulo, 1935Broch.-1 vol.-Doação do Dr. Manuel Augusto Vieira Netto.

Rébora (Juan Carlos)-El Estado de Sitio y la Ley Histórica del Desborde Institucional-La Plata, 1935Broch.-1 vol.-Permuta.

Reforma Judiciaria do Estado de São Paulo-Analyse de discussão do projecto, na Camara dos Deputados, na sessão de 1913-São Paulo, 1914Encad.-1 vol.-Doação do Dr. Azevedo Marques.
Regimen das prisões na America Septentrional-Rio de Janeiro, 1831Broch.-1 vol.-Doação do Prof. Dr. Francisco Morato.

Regulamento da Bibliotheca da Procuradoria Judicial da Fazenda do Estado-São Paulo, 1935-Broch.-1 vol.-Doação do Dr. José Fernandes Moreno.

Regulamento da Faculdade de Medicina da Universidade de São Paulo -São Paulo, 1935-Broch.-1 vol.Permuta.

Riege (Heinz)-Die Gebietshoheit Chinas und ihre Einschränkungen-Breslau, 1933-Broch.-1 vol.-Permuta.

Ripert (Georges)-Le Régime Démocratique et le Droit Civil Moderne -Paris, 1936-Broch.-1 vol.-Compra.

Roceo (Alfredo)-La sentenza civile -Torino, 1906-Broch.-1 vol.-Doação.

Rodrigues de Meréje-Que é a socio logia-São Paulo, 1935-Broch.-1 vol-DDoação do autor.

Rodrigues Doria-O projecto de Codigo Crimlinal Brasileiro-Bahia, 1936-Broch.-1 vol.-Doação do autor.

Rolland (E.)-La loi de réalisation humaine dans Saint Thomas-Paris, 1935-Broch.-1 vol.-Compra.

Rolland (Loais)-Précis de Droit Administratif-Paris, 1928-Encad.-1 vol.-Permutado.

Roquette Pinto-Estudos afro-brasileiros-Rio, 1935-Broch.-1 vol.-Doação da Livraria Universal.

Rotary-Club de Pelotas-Defesa de Pelotas-Pelotas, $\quad$ 1930-Broch -1 vol.-Doação.

Rubio Braziliano-O Rio Grande do Sul e a Cisplatina-Porto Alegre, 1935-Broch.-1 vol.-Doação do autor.

Ruggiero (Roberto de) -Istituzioni di Diritto Civile-Messina-Milano, 1934-Broch.-3 vols.-Compra.

Sainctelette (G.)-L'Assurance-Incencendie pour compte de qui il ap- 
partiendra-Paris, $\quad$ 1910-Broch.-1 vol.-Doação.

Saint-Leon (E. Martin)-Cartells y trusts-Madrid,-Encad.-1 vol.--Permutado.

Schiebel (Heinz)-Die Surrogation nach \& 1381 BGB-Breslat, 1934 Broch.-1 vol.-Doação.

Schlegel (Hans)-Der Eigetumsbegriff in den Enteignungs bestimmungen der Weimarer Verfassung-Berlim, 1934-Broch.-1 vol.-Permuta.

Schwarzer (Norbert)-Der Nachlieferungsanspruch Käufers bei Sachmägeln in deutschen und österreichischen Recht-Breslau, 1934-Broch. -1 vol.-Doação.

Scialoja (Vittorio)--Dizionario pratico di Diritto Privato-Vols. I, II e III-Milano-Broch.-3 vols.-Doação.

Scritti Teorico-Pratici sulla nuova Legislazione Penale Italiana-Bologna, 1932/1933-Broch.-2 vols.Compra.

Seidlitz (Otto)-Uberdie Pflicht einen Notstand zu bestehen-Breslau, 1934 -Broch.-1 vol,--Permuta.

Seybold (Heinz)-Die Geschäftsgrundlage-Breslau, 1934-Broch.-1 vol. -Doação da Universidade de Breslau.

Simon (Raul)-Economia PoliticaSantiago-Chile, 1927-Broch.-3 vols.-Compra.

Skrotzki (Arthur)-Die Novation nach österreichischem und reichsdentschem Recht-Breslau, 1933-Broch. -1 vol.-Permuta.

Solidonio Leite-Desapropriação por utilidade publica-Rio de Janeiro, 1903--Broch.-1 vol.--Doaçãob

Sonderabdrack aus der zeitschrift der Savigny-Stiftung für Rechtsgeschichte-LV Band-Rom. Abt, 1935Broch.-1 vol.-Doação de Dr. Hans Kreller.

Spiegel (Ludwig)-Derecho Administrativo-Barcelona, s/d-Encad.-1 vol.-Compra.

Sraffa (Angelo)-Commentario al Codice di Commercio-Milano-Broch. -8 vols.-Doação.
Stanner (Kurt)-Die Untrene in der Lehre vom zusammentressen mehrerer Gesetzesverletzungen-Breslau, 1934-Broch.-1 vol.-Doação.

Starke (Klans)-Die Völkerrechtssubjektivität der Verwaltungsunionen -Breslau, 1933-Broch.-1 vol.Permuta.

Steinke (Herbert)-Die Donau als Internationale Wasserstrasse-Breslau -Broch.-1 vol.--Permuta.

Sturzo (Luigi)--Essai de sociologieFrance, 1935-Broch.-1 vol.-Compra.

Tarquinio Giglio e Rui Calasans de Araujo-A união da Austria á Allemanha perante a Justiça Brasileira -São Paulo, 1936-Broch.-1 vol.Doação dos autores.

Teixeira (Antonio Ribeiro de Liz)Curso de Direito Civil PortuguezCoimbra, 1856-Encad.-3 vols-Doação.

Teulet (A. F.)-Dictionnaire des Codes Français ou Manuel du DroitParis, 1875-Encad.-1 vol.-Doação.

Thayer (William Roscoe)-Democracy: Discipline: Peace. Cambridge, 1919 -Encad.-1 vol.--Permuta.

Themistocles Brandão Cavalcanti-Do mandado de segurança-Rio de Janeiro, 1934 -Encad.1 vol.-Compra.

Thom (Walter)-Die Eutwicklung der Selbstverwaltung auf dem Gebiet der Wohlfahrtsstege in Preuszen seit dem 9-November 1918-Breslau, 1933--Broch.-1 vol.-Permuta.

Tischer (Sigrid)-Das Vergessen als Fahrlässigkeit-Breslau, 1934 -Broch.-1 vol.-Permuta.

Trabajos de la Catedra de Derecho Administrativo en la Universidad de Oviedo-Oviedo, 1934 -Broch. -1 vol.-Permuta.

Triepel (E.)-Diritto Internazionale e Diritto interno--Torino, 1913-Broch. -1 vol.--Permuta.

Troplong-Commentaire du prèt, du dépost, du séquestre et des contrats aléatoires-Bruxelles, 1845-Encad. 
$\longrightarrow 1$ vol.-Doação do Dr. A. Viriato Pereira.

Truchy (Enrique)-Tratado Elementar de Economia Politica-Madrid. 1935 -Broch.-1 vol.-Doação de "Editorial Reus", S. A.-Madrid.

Un gran triunfo de Colombia-Los derechas de Colombia y la activitud del Gobierna del Perú, juzgado por la Sociedad de las Naciones y los Estados Unidos de America-Madrid, 1933-Broch.-1 vol.-Doação.

Valéry (Jules)-Des Chèques en droit français-Paris, 1936-Broch.-1 vol. -Compra.

Vaquette (Manuels)-La capacité en droit-Paris, 1935/1936-Broch.-2 vols.-Compra.

Venturino (Augustin)-Sociologia General: La interdependencia-Coruña, 1935-Broch.-1 vol.-Doação do autor.

Viamonte (Carlos Sanchez)-El Habeas Corpus la libertad $\boldsymbol{y}$ su garantiaBuenos Aires, 1927-Broch.-1 vol. -Permutado.

Vicente Alves de Paulo Pessoa-Codigo Criminal de Primeira Instancia do Imperio do Brasil-Rio de Janeiro, 1880-Encad.-1 vol.-Doação.

Vidigal (Alcides da Costa)-Accidentes no trabalho-Legislação socialSão Paulo, 1935-Broch.-1 vol.Doação.

Vieira Ferreira-Substitutivo ao projecto de Codigo Commercial-Rio de Janeiro, 1929 -Broch.-1 vol.-Doação do autor.

Villalva (Alfredo Mendizabal)-Concepcion modernas (Conferencias en la Universidad Internacional de Verano en Santander)-Madrid, 1935 -Broch.-1 vol.-Doação do autor.

Viola (G.)-La Costituzione individuale-Bologna. 1933-Broch._2 vols.Compra.

Visconde de Porto Seguro-A questão da Capital: Maritima ou no Interior? Vienna d'Austria, 1877-Broch. -1 vol.-Doação do Dr. Alcides Bezerra.
Vogel (Ulrich))-Die Rechtslage schwimmender Flugstützpunkte-Berlim -Broch.-1 vol.--Permuta.

Vogt (Günther)-Die "Voranssertzung" eine Frage von Trenund Glauben-Breslau, 1934-Broch.-1 vol.-Doação.

Waldemar Ferreira-O casamento religioso de effeitos civis-São Paulo, 1935-Broch.-1 vol.-Doação do autor.

Waldemar Ferreira e Thomaz Lessa - O Pacto de Retrovenda e o Direito de Resgate-São Paulo, 1935 -Broch.-1 vol.-Doação.

Wallanger (Helga)-Das erzwungene Sparen-Breslau, 1930-Broch.-1 vol.-Permuta.

Webel (Wolfgang)-Die Einred des nichterfïlletem Vertrages des $\S 320$ Abs. 1 Satz 1 B g B. und das zurücklehaltungsrecht des $\S 273$ Abs. 1 BGB.-Breslau, 1933-Broch.-1 vol.-Doação.

Walther (Leon)-Orientation professionelles et carrières libérales-Paris, 1936-Broch.-1 vol.-Doação do autor.

Weidner (Willi)-Die strasrechftliche Verawortlickeif der aktienrechflichen Organe nach $\S 312$ H. G. B.-Breslau-Broch.-1 vol.-Permuta.

Welkisch (Kurt)-Der rechtswidrige bindende Dienstbesehl in seiner Abhängigkeit von der jeweilingen Staatsauffassung-Breslau, 1934 Broch.-1 vol.-Permuta.

Wenzel (Carl)-Die Erfüllungsfrage bei einem unter Eigentumsvorbehalt abgeschlossenen Kauf von Sachen Breslau, 1934-Broch.-1 vol.-Doação.

Wolff (Christian)-Jus gentium Methodo Scientifica PertractatumWashington, 1934-Encad.-2 vols.Permuta.

\section{FILOLOGIA (4)}

Antenor Nascentes e Costa MansoSobre a questão orthographica-Rio. 1935-Broch.-1 vol.-Doação. 
Couto de Magalhães-O SelvagemSão Paulo, 1933-Broch.-1 vol.Compra.

Francisco Luiz Pereira-Orthographia usual portugueza em 25 liçõesManáus, 1915-Broch.-1 vol.--Permuta.

J. Norberto de Soiza Silva-Gallicismos, palavras e phrases da lingua franceza introduzidas por descuido ignorancia ou necessidade na lingua portugueza-Rio de Janeiro, 1877Encad.-1 vol.-Doação do Dr. João Rodrigues de Meréje.

Mario Marroquim-A lingua do nordeste (Alagôas e Pernambuco)-São Paulo, 1934 -Broch.-1 vol.-Compra.

Plinio Ayrosa-O caderno da lingua de Fr. Arronches-Vocabulario Portuguez-Tupy-São Paulo, 1935Broch.-1 vol.--Doação do autor.

Renato Mendonça-A influencia africana no portuguez do Brasil-São Paulo, 1935-Broch.-1 vol.-Compra.

\section{CIENCIAS PURAS (5)}

Ameghino (Florentino)-Obras póstumas y truncas-vol. XIX-La Plata, 1935-Broch.-1 vol.-Permuta.

Antonio de Góes-Problema siderurgico brasileiro-Rio de Janeiro, 1935-Broch.-1 vol.-Doação.

Dehn (Edgar)-Algebraie charts-New York, 1930-Encad.-1 vol.-Doação do autor.

Edmundo Franca Amaral-A energia electrica no Brasil-Rio de Janeiro, 1935-Broch.-1 vol-Doação.

Jacob (Max)-Le Phanérogame-Paris, 1918-Broch.— 1 vol.-Doação.

Josué de Castro-Alimentação e raça -Rio de Janeiro, 1936-Broch.-1 vol.-Compra.

Papers from the Geological Departament Glasgow University-vol. XVII

-Glasgow, 1935-Encad.--1 vol.-Permuta.

\section{CIENCIAS APLICADAS (6)}

A aeronantica civil no Brasil-Rio de Janeiro, 1935-Broch.-1 vol.-Doação.

Alexandre Moscoso- -A alimentação dos escolares-Rio de Janeiro, 1935Broch.-1 vol.-Permuta.

Emilio de Maya-A defesa do assucar e o problema do alcool anhydroMaceió, 1935-1 vol.-Doação.

Eugenio Coutinho-Maximas sobre a alimentação-Rio de Janeiro, 1935 -Broch.-1 vol.-Permuta.

Eugenio Coutinho- $O$ dia anti-venereo -Rio de Janeiro, 1935-Broch.-1 vol.-Permuta.

Henrique Roxo-Modernas noções sobre doenças mentaes-Rio-Broch. -1 vol.-Compra.

Keen (W. W.)-Medical Research and Human Welfare-Cambridge-Encad. -1 vol.-Permuta.

Octavio de Freitas-Doenças Africanas no Brasil-São Paulo, 1935-Broch. -1 vol.-Compra.

\section{BELAS ARTES (7)}

Conselho Regional de Engenharia e Architectura da 6." Região-São Paulo, 1936-Broch.-1 vol.-Doação.

Farmer (Herbert)-New MozartianaThe Mozart Relics in the zavertal Collection at the University of Glasgow-Glasgow, 1935-Broch.-1 vol. -Permuta.

\section{LITERATURA (8)}

A de Andrade Silva-Leão $X-O$ scelerado João de Médicis-Tragedia eclesiastica em 1 acto-São Paulo, 1934-Broch.-1 vol.-Doação do Gremio de Cultura Mauá.

Albano Salles-O Chanceller da Paz -Rio, 1935-BrochJ-1 vol.-Doação do Ministerio das Relaçẽes Exteriores. 
Alfaro (Gregorio Araóz)-Discurso pronunciado en el acto inaugural de la Cruzada contra la Tuberculosis -Buenos Aires, 1935-Broch.-1 vol. - Permuta.

Alpheu Domingues-Discurso pronunciado por occasião da inauguração da Estação Experimental do Servico de Plantas Texteis, em Alagoinha, na Parahyba-Rio de Janeiro, 1936-Broch.1 vol.-Doação do autor.

Antonio Constantino--Este é o canto da minha terra...-São Paulo, 1927 -Broch.-1 vol.-Doação do autor.

Appolinaire-Paris-Broch.-1 vol.Doação.

Baptista Pereira-Figuras do Imperio e outros ensaios-São Paulo, 1934 Broch.-1 vol.-Compra.

Chateaubriand (M. le Vicomte de)Mélanges Politiques et Littéraires -Paris, 1845-Encad.-1 vol.-Doaşão.

Despues del atentado-La palabra del Presidente del Brasil Dr. Getulio Vargas a borda de la nave capitana de la Escuadra Brasileña-Broch.1 vol.-Doação.

Jiménez (Don José Gonzáles Menezes y)-Discurso de apertura del Curso Academico de 1935/1936-Sevilla, 1935-Broch.-1 vol.-Permuta.

Mark Twain-Aventuras de HuckSão Paulo, 1934-Broch.-1 vol.Doação.

Paulo Setubal-As maluquices do Imperador-São Paulo, 1926-Broch.1 vol.-Doação de Jamil Miguel Nami.

Recepção a Navarro de Andrade (Discursos)—São Paulo, 1934 -Broch.-1 vol.-Doação.

Rodrigo Octavio-Minhas memorias dos outros-Nova série-Rio de Janeiro, 1935-Broch.-1 vol.-Doação do autor.

Rodrigo Octavio-Minhas memorias dos outros-1. a série-Rio de Janeiro, 1934-Broch.—1 vol.-Doação do autor.
Ruy Barbosa-Mocidade e exilio-São Paulo, 1934-Broch.-1 vol.-Compra

Tristão de Athayde-Estudos. 2. ${ }^{\mathrm{a}}$, 4.* e 5." Série-São Paulo, 1933/1934Broch.-3 vols.-Compra.

\section{HISTORIA E GEOGRAFIA}

A. J. de Sampaio-Biogeographia dynamica-São Paulo, 1935-Broch.-1 vol.-Compra.

A. J. de Sampaio-Phytogeographia do Brasil-São Paulo, 1934-Broch. 1 vol.-Compra.

Affonso Celso-Oito annos de Parlamento-Reminiscencias e notasRio de Janeiro, 1901-Broch.-1 vol. -Doação do Prof. Dr. Francisco Morato.

Affonso de E. Taunay-Visitantes do Brasil Colonial (Seculos XVI XVIII -São Paulo, 1933-Encad.-1 vol.Compra.

Alberto Rangel--D. Pedro Primeiro e a Marqueza de Santos-França, 1928 -Broch.-1 vol.-Compra.

Alcides Bezerra-Sylvio Romero o pensador e o sociologo-Rio de Janeiro, 1935-Broch.-1 vol.-Doação do autor.

Alfredo Ellis Junior-O Bandeirismo Paulista e o recúo do meridiano -São Paulo, 1934-Broch.-1 vol.Compra.

Alfredo Ellis Junior-Populaçóes paulistas-São Paulo. 1934-Broch.-1 vol.-Compra.

Angyone Costá-Introducção á Archeologia Brasileira-São Paulo, 1934Broch.-1 vol.-Compra.

Antonio Gontijo de Carvalho-Calogeras-São Paulo, 1935-Broch.-1 vol. -Compra.

Augusto de Saint Hilaire-Segunda viagem do Rio de Janeiro a Minas Geraes e a São Paulo (1822)-São Paulo, 1932-Broch.-1 vol.-Compra.

Aureliano Leite-Martyrio e Gloria de São Paulo-São Paulo, 1934-Broch. -1 vol.-Doação de Jamil Miguel Nami. 
Baptista Pereira-Vultos e episodios do Brasil-São Paulo-Broch.-1 vol. -Compra.

Brasilio de Magalhães-Expansão Geographica do Brasil Colonial-São Paulo, 1935-Broch.-1 vol.-Compra.

Bruno Lobo-Esquecendo os antepassados-Combatendo os extrangeiros -Rio de Janeiro, 1935-Broch.-1 vol.-Doação de Flavio Mendes.

C. de Mello Leitão-Visitantes do primeiro Imperio-São Paulo, 1934Broch.-1 vol.-Compra.

Cantinho Filho-Os bachareis de 1904 -Reminiscencias-Faculdade de Direito de São Paulo-São Paulo, 1934 -Broch.-1 vol.-Doação do autor.

Commemoração do 382.0 anniversario da fundação de São Paulo-Rio de Janeiro, 1936-Broch.-1 vol.-Doação do Centro Paulista.

Decada Republicana-Vols. I ao VI e VIII-Rio de Janeiro, 1900/1902Encad.-7 vols.-Permuta

E. Roquette Pinto-Rondonia-São Paulo, 1935-Broch.-1 vol.-Compra.

El Circulo de Armas en el cincuennario de su fundación $1885 / 1935-$ Buenos Aires, 1935-Broch.-1 vol. -Permuta.

Ernesto de Souza Campos-Japão visto atravez de uma viagem ao Oriente realizada por Universitarios da Faculdade de Medicina de São Paulo-São Paulo, 1935-Broch.-1 vol.-Doação.

Esquisse de son histoire-Revue de l'Institut Historique et Géographique du Brésil-Rio de Janeiro, 1928 -Broch.-1 vol.-Permuta.

Expilly (Charles)-Mulheres e costumes do Brasil-São Paulo, 1935Bro.ch-1 vol.-Compra.

Exposição preparatoria industrial e historica Farroupilha em 30 de Junho de 1935, em homenagem ao primeiro centenario da elevação de Pelotas á cathegoria de CidadeBroch.-1 vol.-Doação do Gremio de Cultura Mauá.
Finzi (Marcello)-Max Alsberg-Cittả di Castello, 1934-Broch.-1 vol. Doação do autor.

Frederico A. Rondon-Pelo Brasil Central一São Paulo, 1934-Broch.-1 vol. -Compra.

Gastão Goulart-Verdades da revolução paulista-1933-Broch.-1 vol. -Doação de Jamil Miguel.

Guerra da Europa-Documentos Diplomaticos-Attitude do Brasil-Ministerio das Relações Exteriores-Rio de Janeiro, 1918-Broch.-1 vol.Doação.

Gustavo Rarroso-Historia Militar do Brasil-São Paulo, 1935-Broch.-1 vol.-Compra.

Homenaje a la memoria de Juan Vucetich-Buenos Aires, 1935-Broch. -1 vol.-Permuta.

I. Arton et Albert Blanc-Oenvre Parlamentaire du Comte de Cavour -paris, 1862-Encad.-1 vol.-Doação do Prof. Dr. Francisco Morato

J. F. de Almeida-Primeiros povoadores do Brasil 1500/1530-São Paulo, 1935-Broch.-1 vol.-Compra.

La obra de Colombia em su territorio amazónico-Madrid, 1932-Broch.-1 vol.-Doação da Bibliotheca Riograndense.

La Biblioteca de la Facultad de Ciencias Economicas, Comerciales y Politicas de Rosario al cumplir veinticinco años desde que el poder Ejecutivo Nacional la declaró publica -Santa-Fé, 1935-Broch.-1 vol.Permuta.

Luis da Camara Cascudo-O Conde D'Eu-São Paulo, 1933-Broch.-1 vol.-Compra.

Mac-Pherson (M. J. Gornes)-Venezuela Grafica 1929/1930-Caracas, 1930-Broch.-1 vol.-Doação do autor.

Manoel Bomfim-O Brasil-São Paulo, 1935-Broch.-1 vol.-Compra.

Manuel Osorio-A guerra de São Paulo -São Paulo, 1932-Broch.-1 vol.Doação. 
Mario Travassos-Projecção continental do Brasil-Sâo Paulo, 1935Broch.-1 vol.-Compra.

Maurois (André)-Byron-Paris, s/d. -Broch.-1 vol-Compra.

Max Fleiuss-Apostilas de Historia do Brasil--Revista do Instituto Historico e Geographico Brasileiro-Volume especial-Rio de Janeiro, 1933 -Brch.-1 vol.-Permuta.

Max Fleiuss-Oliveira Lima-Conferencia-Rio de Janeiro, 1928-Broch. -1 vol.-Permuta.

Max Fleiuss-Paginas de Historia-2." edição-Rio de Janeiro, 1930-Broch. -1 vol,-Permuta.

Max Fleiuss-Um marinheiro moderno (Antonio Coutinho Gomes Pereira)-Conferencia-Rio de Janeiro 1927-Broch.-1 vol.-Permuta.

o Centenario da emancipação de Alagôas-Maceió, 1919-Broch.-1 vol.Doação do Dr. Arthur Barreto de Aguiar.

Ouro-Preto-Revista do Instituto Historico e Geographico BrasileiroRio de Janeiro, 1931-Broch.-1 vol. -Permuta.

Pandiá Calogeras-Da Regencî́a á queda de Rozas-São Paulo, 1933Broch-1 vol.-Compra.

Prevost-Paradol-Quelques pages d'Histoire contemporaine--Paris, 1866Broch.-1 vol.-Doação do Prof. Dr. Francisco Morato.
Quarto Centenario da fundação de São Vicente-Revista do Instituto Historico e Geographico Brasileiro -Rio de Janeiro, 1932-Broch.-1 vol.-Permuta.

Revista do Instituto do Ceará-Tomo XLIX-Ceará, 1935-Broch.1 vol.Permuta.

Revista do Instituto Geographico e Historico da Bahia-Vol. LI-Bahia, 1935-Broch.-1 vol.-Permuta.

Revista do Instituto Historioo e Geographico Brasileiro--Vols. 165 e 166 -Tomo especial-Rio de Janeiro, 1920/1933/1935-Broch.-3 vols-Permuta.

Tri-centenario de Cametá 1635/1935Estado do Pará, 1935-Broch.-1 vol. -Doação da Imprensa Official do Estado do Pará.

Urbino Vianna-Bandeira e sertanistas bahianos-São Paulo 1935Broch.-1 vol.-Compra.

Visconde de Taunay-Pedro II-São Paulo, 1933-Broch.-1 vol.-Compra.

Zweig (Stefan)-Erasme-Paris, s/d. -Broch.-1 vol.-Compra.

Zweig (Stefan)-Joseph Fouché-Paris, s/d.-Broch.-1 vol.-Compra.

Zweig (Stefan)-Marie AntonietteParis,-Broch.-1 vol.-Compra.

Zweig (Stefan)-Marie Stuart-Paris -Broch.-1 vol.-Compra. 